\title{
EDITORIAL
}

\section{Bildungsforschung mit Daten der amtlichen Statistik}

\section{Educational Research with Data of Official Statistics}

Die empirische Bildungsforschung hat in den letzten beiden Jahrzehnten einen bemerkenswerten Aufschwung erfahren. Vor allem die Schulleistungsforschung, die Forschung zu den Bedingungen von Lernprozessen und gelingendem Unterricht hat durch die Leistungsvergleichsstudien auf internationaler und nationaler Ebene erheblich zugenommen und das Wissen in diesen Bereichen deutlich verbessert. Durch das Nationale Bildungspanel (NEPS) wurde für die Analyse von Bildungsverläufen eine breite und intensiv von der Forschung genutzte Datenbasis geschaffen.

Mit dem Nationalen Bildungsbericht und den Bestrebungen zu länderspezifischen und kommunalen Bildungsmonitorings wurde verstärkt auch der Datenbestand der amtlichen Bildungsstatistik für überwiegend deskriptive Studien herangezogen. Nur in sehr geringem Umfang wurde der vorhandene Datenbestand bislang für analytische Auswertungen im Rahmen von Monitoringvorhaben oder für eine flankierende Forschung verwendet. Die im letzten Jahrzehnt durch ein erweitertes Datenangebot und eine verbesserte Datenbereitstellung gebotenen Möglichkeiten der amtlichen Bildungsstatistik sind daher bisher kaum erschlossen. Zu den Verbesserungen im Bereich der Bildungsstatistiken gehören vor allem die Kinder- und Jugendhilfestatistik seit 2006, die von der Kultusministerkonferenz beschlossene Einführung einer Individualstatistik für den Schulbereich, die neue Statistik zur betrieblichen Berufsausbildung und die wieder eingeführte Möglichkeit, die Hochschulstatistik für die Analyse von Bildungsverläufen zu nutzen. Diese Möglichkeit über eine anonymisierte Personenkennung bieten inzwischen auch die Schulstatistiken einiger Länder.

Warum die amtliche Bildungsstatistik relativ wenig durch die Bildungsforschung genutzt wird, ist vermutlich auf mehrere Gründe zurückzuführen: Eine Ursache für die geringe Nutzung der Bildungsstatistiken durch die Forschung könnte zunächst eine fehlende Berücksichtigung der Nutzungsmöglichkeiten für Sekundäranalysen in der Lehre sein. Nur in wirtschaftswissenschaftlichen Studiengängen wird das Datenangebot der amtlichen Statistik vermittelt und der Umgang mit dem Datenmaterial der amtlichen Statistik für Sekundäranalysen auch gelehrt. Eine weitere Ursache könnten geringe Kenntnisse über die geregelten Zugänge zu dem Kernbestand der 
Daten sein. Jedoch stehen differenzierte - wenn vielfach auch noch unzureichende - Informationen zum Datenangebot der amtlichen Bildungsstatistik zur Verfügung (URL: https://www.bildungsserver.de/Gesamtstatistiken-zur-Bildung-in-Deutschland299-de.html). Schließlich könnten auch Zugangsbeschränkungen oder besondere Zugangshürden dazu beitragen, dass die Daten nicht in relevantem Umfang genutzt werden (vgl. Bundesministerium für Wirtschaft und Energie, 2016).

Wegweisend für die Bereitstellung von schulischen Individualdaten für Monitoringund Forschungszwecke ist Hamburg. Mit dem neu ins Schulgesetz aufgenommenen Paragraphen 98a (Vertrauensstelle) besteht eine gesetzliche Grundlage, um die in großem Umfang vorhandenen schulstatistischen sowie beispielsweise Leistungsdaten (KERMIT: Kompetenzen ermitteln) sowohl für anspruchsvolle Monitorings im Querund Längsschnitt miteinander verknüpfen als auch für wissenschaftliche Untersuchungen bereitstellen zu können (vgl. URL: http://www.schulrechthamburg.de/ jportal/portal/t/1deo/bs/18/page/sammlung.psml/action/controls.sammlung.Change Werknavigation? nid=3u\&nac $=$ select $\&$ showdoccase $=1 \&$ doc.id $=$ jlr-SchulGHAV32P98 a\&doc.part=S).

Ein Beispiel für die ertragreiche Kooperation zwischen Wissenschaftler*innen und dem Institut für Bildungsmonitoring und Qualitätsentwicklung (IfBQ) der Behörde für Schule und Berufsbildung stellt das Evaluationsvorhaben EiBiSch (Evaluation inklusiver Bildung in Schulen) dar, bei dem neben von den Wissenschaftler*innen erhobenen Daten schulstatistische und Leistungsdaten im Quer- und Längsschnitt miteinander verknüpft worden sind (zur Datenverknüpfung siehe Fickermann \& Doll, 2015, zum Abschlussbericht des Vorhabens Schuck, Rauer \& Prinz, 2018).

Die bislang in einigen Ländern eher zögerliche Einführung des von ihnen in der KMK mitbeschlossenen Kerndatensatzes erfährt aktuell durch zwei bildungspolitische Diskussionen wieder verstärkte Aufmerksamkeit: Zum einen arbeiten die Länder als Reaktion auf die von den Koalitionspartnern im Koalitionsvertrag verabredete Etablierung eines nationalen Bildungsrates am Entwurf eines eigenen Bildungsstaatsvertrages, der auch Passagen zur Weiterentwicklung der Schulstatistik enthalten soll. Zum anderen hat der Nationale Normenkontrollrat in einem Gutachten die Einführung eines registerbasierten Zensus angeregt, bei dem auch bildungsstatistische Daten genutzt werden sollen (2017). Das Statistische Bundesamt hat in einer Vorstudie hierzu erste Überlegungen zur Einführung eines nationalen Bildungsregisters angestellt, die zwischen Bund und Ländern kontrovers diskutiert werden, da die Länder dabei ihre Zuständigkeit für den Schulbereich und damit auch für die Schulstatistik unzureichend gewahrt sehen.

Das Bundesministerium für Bildung und Forschung (BMBF) hat $\mathrm{zu}$ den rechtlichen Rahmenbedingungen für ein nationales Bildungsregister ein Gutachten an 
Prof. Mario Martini von der Verwaltungshochschule Speyer vergeben, in dem dieser sich für einen Bildungsstaatsvertrag zwischen Bund und Ländern ausspricht und die von der Länderseite vorgebrachten ersten Überlegungen zur Einschaltung eines vertrauenswürdigen Dritten (Trusted Third Party) aufgreift (Martini et al., 2019). Gleichzeitig arbeitet das Statistische Bundesamt im Auftrag des BMBF an einer Machbarkeitsstudie für ein nationales Bildungsregister. Ob und in welchem Maße die Interessen der Länder dabei berücksichtigt werden, ist derzeit noch unklar. Die mit amtlichen Daten empirisch arbeitenden Bildungsforscherinnen dürften jedoch größtes Interesse an der Etablierung eines nationalen Bildungsregisters mit wissenschaftlichen Nutzungsmöglichkeiten der Registerdaten haben. Insofern ist nur zu wünschen, dass sich Bund und Länder auf eine Lösung verständigen, die die Datenhoheit der Länder und die wechselseitigen Interessen von Bund und Ländern berücksichtigt.

Absicht des vorliegenden Beiheftes ist es, die vielfältigen Analysemöglichkeiten von Daten der amtlichen Statistik zu verdeutlichen und auf spezifische Fragestellungen hinzuweisen, die nur mit diesem Material zu bearbeiten sind. Üblicherweise wird die amtliche Statistik deskriptiv (z.B. im Rahmen von Monitoringverfahren) eingesetzt, um beispielsweise die Entwicklung von Schülerzahlen, regionale Unterschiede des Angebots von Kindergartenplätzen, die Qualifikation des Personals oder die Erreichung eines Ausbauziels zu dokumentieren. Zeitvergleiche, Regionalvergleiche und kriteriale Vergleiche bilden die Grundlagen von Auswertungen statistischer Daten. In Forschungsvorhaben mit amtlichen Daten wird darüber hinaus auch eine analytische Absicht verfolgt. Grundlegende Fragestellungen richten sich auf die mit dem Bildungsangebot erreichte Versorgung und die sachgerechte Verteilung des Angebots und dessen Personalausstattung (Daseinsvorsorge). Aus der Sicht der Bildungsteilnehmer ${ }^{*}$ innen werden die Bildungsmöglichkeiten und die Zugänglichkeit von Angeboten untersucht. Unterschiede in den Angeboten und deren Nutzung nach sozialen Gruppen, Geschlecht, Nationalität, Migrationserfahrung und anderen Ungleichheitsdimensionen sind weitere zentrale Untersuchungsabsichten. Eine zusätzliche Analyseperspektive eröffnen die Daten der Bildungsfinanzstatistik in der Kombination mit Teilnehmer*innen- oder Personaldaten.

Deutlich wird, wie breit gefächert die Datenbasis ist und wie vielfältig die Analyseperspektiven sein können, die über eine Verknüpfung der Daten untereinander und mit anderen Daten noch zusätzlich erweiterbar sind. Grundsätzlich existieren statistische Informationen über

- die Einrichtungen nach Struktur und Trägern,

- die Besucherinnen der Einrichtungen (Kindergartenkinder, Schülerinnen, Auszubildende, Hochschüler*innen) nach individuellen Merkmalen,

- den Sozialraum, in dem die Einrichtung liegt, und den Sozialraum, in dem die Besucher*innen der Einrichtungen wohnen,

- die Art und fachliche Struktur des Angebots, 
- das Personal nach persönlichen Merkmalen, Qualifikation und Beschäftigungsumfang und

- die öffentlichen Ausgaben.

Im Weiterbildungsbereich beschränkt sich dieses Informationsangebot auf die Volkshochschulen.

Angesichts der Vielfalt der Möglichkeiten kann auch dieser Sammelband nur einen begrenzten Einblick in mögliche Forschungsperspektiven geben. Die Beiträge stammen schwerpunktmäßig aus dem Schulbereich und konzentrieren sich auf die Einrichtungen und die Teilnehmer*innen. Finanzstatistische Analysen existieren bisher kaum und fehlen auch in diesem Band. Aber auch zum Personal im Bildungswesen fehlt ein Beitrag.

$\mathrm{Zu}$ Verknüpfungen von Daten der IQB-Schulleistungserhebungen mit Daten der Schulstatistik in Hamburg und Bremen liegen noch keine Ergebnisse vor. Zu erwarten sind interessante Aufschlüsse - sowohl bezogen auf den Aussagegehalt der schulstatistischen Informationen als auch den der vom IQB eingesetzten Kontextfragebögen. Eine Verknüpfung schulstatistischer Daten mit Leistungsinformationen ist sonst nur möglich, wenn die Forscher*innen selbst die Daten erheben (wie in dem Beitrag von Hogrebe \& Pomykaj), ein Zugang zu den VERA-Daten ermöglicht wird (Thoren, Hannover \& Brunner) oder eine Kooperation zwischen Forscher*innen und dem zuständigen Kultusministerium vereinbart wird, was außerhalb Hamburgs bisher kaum gelang.

Durch die hier vorgestellte Darstellung der methodischen Ansätze und der erzielten Ergebnisse sollen Wissenschaftler*innen Anregungen für eigene Forschungsarbeiten erhalten und motiviert werden, in größerem Maße als bisher Daten der amtlichen Statistik für eigene Forschungsarbeiten zu nutzen, auch in Kombination mit selbst durchgeführten Erhebungen. Den datenhaltenden Stellen in den für die verschiedenen Bildungsbereiche zuständigen Ministerien oder Behörden und in den Statistischen Ämtern des Bundes, der Länder und der Kommunen sollen die in dem Beiheft vorgestellten Projekte den Wert ihrer Daten für wissenschaftliche Auswertungen verdeutlichen, um auf diese Weise ein Bewusstsein für die Notwendigkeit eines leichteren Datenzugangs zu schaffen. Zugleich soll auch der Wert von Ergebnissen von Forschungsvorhaben mit amtlichen Daten für Steuerungsentscheidungen verdeutlicht werden, die durch einfache deskriptive Auswertungen nicht zu erzielen wären.

Eingeleitet wird der Sammelband durch einen Text von Mitgliedern des Rats für Sozial- und Wirtschaftsdaten (Artelt, Bug, Kleinert, Maaz \& Runge) zum Stand der Bemühungen, den Forschungszugang zu Daten der amtlichen Bildungsstatistik über Forschungsdatenzentren auszubauen und Zugangshindernisse abzubauen. 
In einem zweiten Text zur Datenlage und zum Datenzugang befasst sich Mundelius mit dem Stand der Umsetzung des Kerndatensatzes der Kultusministerkonferenz, auf den diese sich schon vor fast 15 Jahren einigte. Die Schulen und damit die Schulstatistik bilden zwar den Kernbereich des Bildungswesens. Die Schulstatistik ist aber keine amtliche Statistik auf einer bundesweit einheitlichen gesetzlichen Grundlage, wie im Vorschulbereich, bei der Berufsbildung und im Hochschulbereich, sondern eine Behördenstatistik der Kultusministerien der Länder. Zum einen sind die rechtlichen Grundlagen zwischen den Ländern sehr unterschiedlich; zum anderen ist auch der Forschungsdatenzugang nicht vergleichbar mit $\$ 16$ Abs. 6 des Bundesstatistikgesetzes geregelt, der auch den Zugang zu Einzeldaten für Forschungszwecke gestattet. Für die Bundesstatistik zum Schulbereich und die internationalen Datenanforderungen der EU, der UN und der OECD gibt es ebenfalls keine rechtlichen Regelungen, die eine für die Bundesrepublik einheitliche Qualität der Datenlieferungen der einzelnen Bundesländer garantieren. Deshalb ist das Bemühen um eine den nationalen und internationalen Erfordernissen angemessene Schulstatistik von erheblicher Bedeutung (siehe hierzu auch die Ausführungen zur Etablierung eines nationalen Bildungsregisters oben).

Nicht realisiert werden konnte die Absicht, einen Beitrag zu den rechtlichen Grundlagen des Zugangs der Forschung zu den Daten der Schulstatistik aufzunehmen, weil zu dieser speziellen Frage keine Autorin bzw. kein Autor gefunden werden konnte. Doch ist zu hoffen, dass das gegenwärtig im Auftrag der Bertelsmann Stiftung erstellte Gutachten zu den Auswirkungen der Informationsfreiheitsgesetze in den Ländern auf den Datenzugang für die Forschung dazu beiträgt, einige offene Fragen zu klären und die Position der Forschung gegenüber häufig wenig auskunftsfreudigen Kultusministerien zu verbessern.

Die Reihenfolge der Beiträge folgt dem Bildungsverlauf. Sie beginnt mit einem Beitrag aus der kommunalen Stadtforschung, der unterschiedliche Datenquellen heranzieht, um die Bedingungen des Aufwachsens in einer Kommune kleinräumig und unter Einbeziehung von Individualdaten zu analysieren. Groos \& Kersting können mit dem verfügbaren Material individuelle Risikofaktoren und schwierige sozial-regionale Förderkonstellationen identifizieren, auf die kommunales Handeln reagieren kann.

Der zweite Beitrag verwendet die Daten der medizinisch-sozialpädiatrischen Schuleingangsuntersuchung und kombiniert sie mit eigenen Erhebungen und den Daten der Kindergartenstatistik. Hogrebe \& Pomykaj interessiert vor allem die Abhängigkeit der Sprachkompetenz von Kindergartenkindern zum Schulbeginn von unterschiedlichen Kontextfaktoren.

Die zwei folgenden Beiträge haben Entwicklungen der Grundschule zum Thema: Kramer \& Bauer analysieren aus bildungsgeografischer Sicht die Entwicklung der Grundschulen in Baden-Württemberg. Die Entwicklung der Grundschulstandorte 
in den neuen Bundesländern mit Blick auf die Folgen von Schulschließungen und die Bedeutung von Privatschulen werden von Helbig, Konrad \& Nikolai analysiert. Zusätzlich beziehen sie in ihre Analysen auch die Schulen der Sekundarstufe unter der Fragestellung ein, wie sich - angesichts des starken Schülerrückgangs - Schulen ohne Sekundarstufe II neben Gymnasien und Schulen mit gymnasialer Oberstufe behaupten konnten.

Zwei Beiträge befassen sich auf der Basis von Daten der Berliner Schulstatistik mit der Auswirkung unterrichtsorganisatorischer Maßnahmen in der Grundschule: Ziegler, Richter \& Hartung-Beck gehen der Frage nach, ob die Entwicklung der Häufigkeit fachfremden Unterrichts mit Merkmalen der Schülerzusammensetzung zusammenhängt, und Thoren, Hannover \& Brunner kombinieren schulstatistische Daten mit VERA-Leistungserhebungen. Sie gehen der Frage nach, ob sich jahrgangsübergreifendes Lernen in der Eingangsphase der Grundschule in ethnisch heterogenen Schulen positiv auf den Leistungsstand der Schülerinnen auswirkt.

Am Beispiel von Nordrhein-Westfalen und Bremen werden in zwei Beiträgen die sozialräumlichen Unterschiede zwischen den Grundschulen untersucht: Jeworutzki \& Schräpler berichten zentrale Ergebnisse aus einer umfangreichen Studie, in der sie Daten der Volkszählungen seit 1961 mit Ergebnissen der Schulstatistik kombiniert haben. Die Unterschiede in den Übergangsquoten zum Gymnasium werden herangezogen, um die Auswirkungen sozialer Segregation auf Bildungschancen zu verdeutlichen. Makles, Schneider \& Terlinden verwenden Bremer Schülerindividualdaten. Sie interessieren das Ausmaß und die Entwicklung der Segregation im Zeitverlauf. Zusätzlich werden von ihnen die Auswirkungen der freien Grundschulwahl auf Segregationsprozesse analysiert.

Der zeitlichen Entwicklung der Nachfrage nach weiterführenden Schulen widmet sich der Beitrag von Neumann \& Maaz, die ebenfalls Daten für Bremen heranziehen. Das verwendete Datenmaterial gestattet es, neben Merkmalen der Schülerzusammensetzung auch Leistungsdaten zu berücksichtigen.

Der zunehmende Wettbewerb zwischen Gymnasien und die Folgen dieses Wettbewerbs sind Thema des Beitrags von Forell, Bellenberg \& im Brahm. Mit einzelschulischen Daten der Schulstatistik lassen sich Informationen über die Standortkonkurrenz, den Zugang zu den Gymnasien, die Durchlässigkeit und die Wahrscheinlichkeit des Verbleibs an den Gymnasien ermitteln, die den Ausgangspunkt der Analysen bilden.

Die letzten beiden Beiträge befassen sich mit der sonderpädagogischen Förderung bzw. der Umsetzung der Inklusion. Kemper \& Goldan analysieren die über die Schulstatistik für Nordrhein-Westfalen verfügbaren Indikatoren und ergänzen sie um die Quote der Inklusionsschulen. Anhand ihrer Analysen werden große regio- 
nale Disparitäten bei der Umsetzung der schulischen Inklusion deutlich. Weishaupt untersucht am Beispiel Hessens die Situation der sonderpädagogischen Förderung in Förder- und allgemeinen Schulen insgesamt und kann dadurch das Zusammenspiel von Förderschule und Inklusion bei unterschiedlichen Angebotskonstellationen darstellen.

Als Herausgeber des Bandes sind wir einerseits dankbar für die sehr interessanten und an vielfältigen Aspekten reichen Beiträge, die wir in diesem Band versammeln können. Dafür danken wir allen Autor*innen und den Gutachter*innen der Beiträge. Andererseits ist es bedauerlich, dass wir keine Beiträge zu den beruflichen Schulen oder $\mathrm{zu}$ den Hochschulen aufnehmen konnten. Unter regionaler Perspektive fällt auf, dass die jährlich veröffentlichte, allgemein zugängliche und sehr differenzierte Schulstatistik von Nordrhein-Westfalen offensichtlich auch Forscher*innen animiert, sie für Querschnitts- oder Quasi-Längsschnittuntersuchungen zu verwenden. Fünf der zwölf Artikel dieses Sonderhefts beziehen sich auf Nordrhein-Westfalen. In den meisten anderen Ländern werden nur wenige Informationen, die über die bundesstatistischen Veröffentlichungen hinausgehen, jährlich veröffentlicht. So ist es kaum verwunderlich, dass nur wenige Beiträge zu anderen Flächenländern Eingang in den Sammelband fanden, obwohl teilweise auf Anfrage sehr differenziertes schulstatistisches Datenmaterial vorliegt. In den Ländern, die den Kerndatensatz der KMK oder zumindest Teile davon bereits umgesetzt haben, wären auch im Längsschnitt verknüpfbare Individualdaten verfügbar. Informieren kann man sich über das potenziell verfügbare Datenangebot über den von der Kultusministerkonferenz beschlossenen Kerndatensatz (URL: http://www.kmk.org/fileadmin/pdf/Statistik/FAQ_KDS. pdf). Insofern hoffen wir, mit diesem Band auch Anstöße dahingehend zu geben, den verfügbaren Datenbestand zu interessierenden Fragestellungen bei den jeweiligen Statistischen Landesämtern bzw. Kultusministerien zu erfragen. Damit verbinden wir die Hoffnung auf zahlreiche weitere Studien, die über die Nutzung der in den Ländern vorhandenen Daten entstehen.

Detlef Fickermann \& Horst Weishaupt

https://doi.org/10.31244/dds.bh.2019.14.01

\section{Literatur und Internetquellen}

Bundesministerium für Wirtschaft und Energie (Hrsg.). (2016). Mehr Transparenz in der Bildungspolitik. Gutachten des Wissenschaftlichen Beirats beim Bundesministerium für Wirtschaft und Energie. Berlin. Zugriff am 06.03.2019. Verfügbar unter: https://www. bmwi.de/Redaktion/DE/Publikationen/Ministerium/Veroeffentlichung-Wissenschaft licher-Beirat/wissenschaftlicher-beirat-mehr-transparenz-in-der-bildungspolitik.pdf? _ blob=publicationFile\&v=7 . 
Fickermann, D., \& Doll, J. (2015). Potential und Technik der Verknüpfung von Befragungsdaten mit schulstatistischen Individualdaten und Leistungsdaten im Projekt EiBiSch. Die Deutsche Schule, 107 (4), 365-374. Zugriff am 06.03.2019. Verfügbar unter: https:// www.waxmann.com/index.php?eID=download\&id_artikel=ART101810\&uid=frei.

Martini, M., unter Mitarbeit von T. Kienle, D. Wagner, Q. Weinzierl \& M. Wenzel (2019). Rechtliche Rahmenbedingungen für ein nationales Bildungsregister. Rechtsgutachten im Auftrag des Bundesministeriums für Bildung und Wissenschaft. Speyer: Deutsche Universität für Verwaltungswissenschaften.

Nationaler Normenkontrollrat (Hrsg.). (2017). Mehr Leistung für Bürger und Unternehmen: Verwaltung digitalisieren. Register modernisieren. NKR-Gutachten 2017. Berlin: NKR. Zugriff am 06.03.2019. Verfügbar unter: https://www.normenkontrollrat.bund.de/ nkr-de/service/publikationen/gutachten/mehr-leistung-fuer-buerger-und-unterneh men-verwaltung-digitalisieren-register-modernisieren--371266.

Schuck, K. D., Rauer, W., \& Prinz, D. (Hrsg.). (2018). EiBiSch - Evaluation inklusiver Bildung in Hamburger Schulen. Quantitative und qualitative Ergebnisse (HANSE - Hamburger Schriften zur Qualität im Bildungswesen, Bd. 17). Münster et al.: Waxmann. Zugriff am 06.03.2019. Verfügbar unter: https://www.waxmann.com/?eID=texte\&pdf=3922Voll text.pdf\&typ=zusatztext.

Statistisches Bundesamt (Hrsg.). (2018). Einrichtung eines Bildungsregisters in Deutschland Vorstudie. Wiesbaden: Statistisches Bundesamt (unveröffentlicht). 